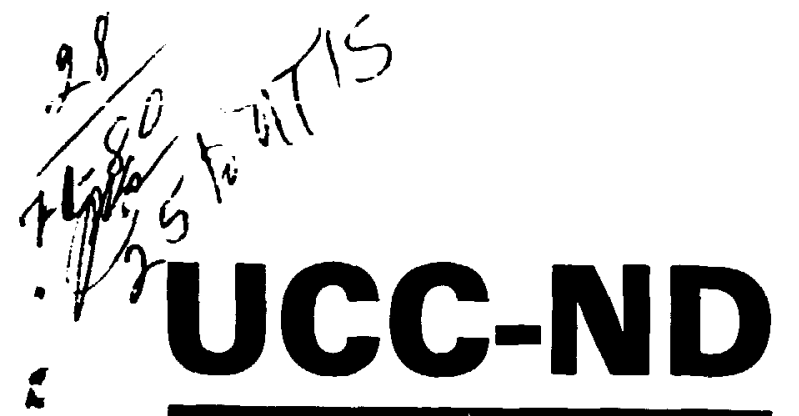

NUCLEAR DIVISION
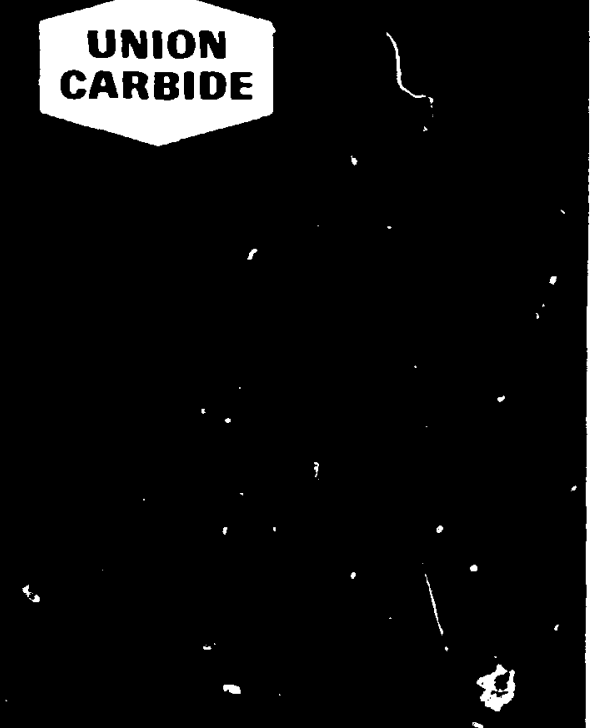

\section{OPERATEO BY}

UNION CARBIOE CORPOPATION

FOR-THE UNITED STATES

DEPARTMENT OF-ENERGY

\section{Conversion and Improvement of the Rutherford Laboratory's Magnetostatic Computer Code GFUN3D to the NMFECC CDC 7600}


ORNL/CSD/TM-108

\title{
CONVERSION AND IMPROVEMENT OF THE FUTHERFOPD LABORATORY'S \\ MAGNETOSTATIC COMPUTER CODE GFUN3D TO THE MMFCC CDC 7600
}

\author{
T. C. Tucker
}

Sponsor: Y-K. M. Peng

Date Published: June 1980

\author{
COMPUTER SCIENCES DIVISION \\ at \\ Oak Ridge National Laboratory \\ Post Office Box $X$ \\ Oak kidge, Iennessee 37830
}

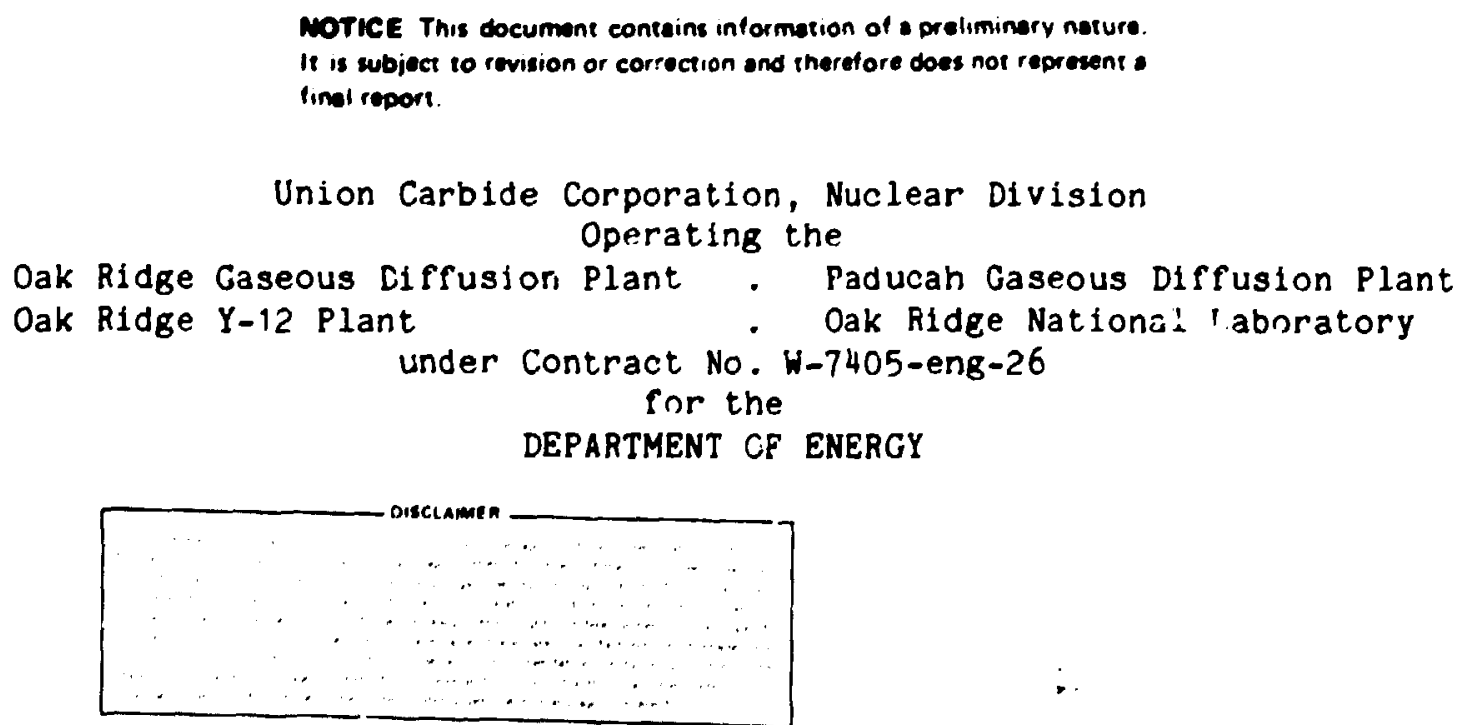

MOTice This document conteins informstion of a preliminery noture.

It is subject to revision or correction and therefore does not represent o

linal report.

Union Carbide Corporation, Nuclear Division Operating the

Oak Ridge Gaseous Liffusion Plant - Paducah Gaseous Diffusion Plant

Oak Ridge Y-12 Plant - Oak Ridge Nations: rabnratory

under Contract No. W-7405-eng-26

for the

DEPARTMENT CF ENERGY 
TABLE OF CONTENTS

ACKNOWLEDGMENTS ....................... v

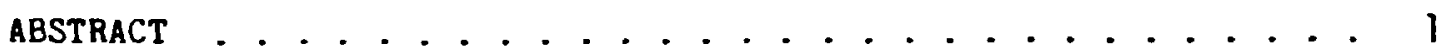

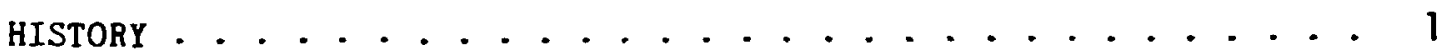

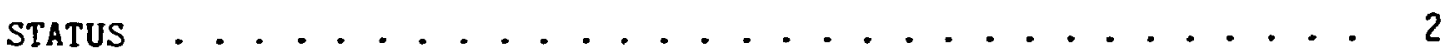

MECHANICS ........................ 3

DIFFERENCES IN GFUN3D ON NMFECC 7600 AND ORNL $360 \ldots 3$

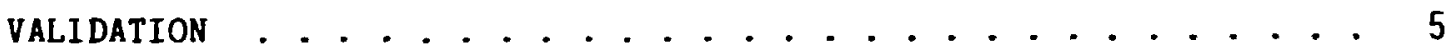

GFUN3D ON THE MMFE NETWORK . . . . . . . . . . . . . 6

SUGGESTIONS FOR FUTURE WORK . . . . . . . . . . . . . . 6

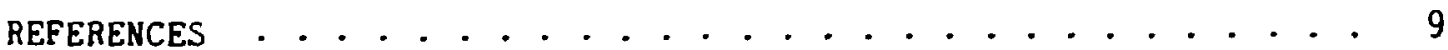

APPENDIX A - GFUN3D NMFE 7600 USER'S GUIDE ........... 11

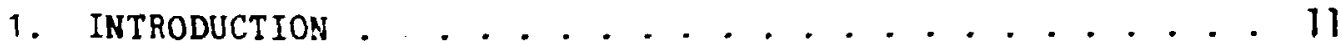

2. INTEGRAL EQUATION METHOD .............. 11

3. THE USE OF INTERACTIVE GRAPHICS . . . . . . . . . . . . il

4. STRUCTURE OF THE GFUN3D PROGRAM ........... 12

5. UNITS . . . . . . . . . . . . . . . 12

6. COMMANDS AND PARAMETERS; TYPES,

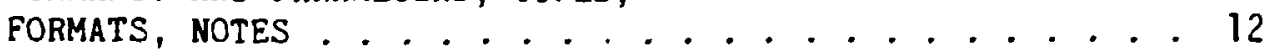

6.1. Command Names: List of Commands ......... 12

6.2. DRAW: Enter Element DATA ......... 13

6.2.1. General Comments.......... 13

6.2.2. DRAW Command for Specifying Coils .... . 13

6.2.?. DRAW Command for Specifying Iron ..... . 13

6.3. MODI and MOVE: MOdify and Move Elements...... 13

6.4. ERASE: Erase Elements ............ 13

6.5. PEC.: Reconstructs Picture ......... 14

6.6. BHDA: Enter B-H Data ............. 14

6.7. GETM: Magnetization Frocedure......... 14

6.8. GETB .................. 16

6.9. MIP................... . . 16

6.10. HARM ................ 16

6.11. File Managament Commands ............. . le

6.12. TFACK: Tracking of Charged Particie3 . . . . . 18

6.i3. GETF: Magnetic Forses ........... 18

6.14. INTE: Integration of Fleld Functions ...... 18 
6.15. FIXI .................. 18

6.16. Other Commands........... 19

?. OFF-LINE GETM . . . . . . . . . . . . . . 19

8. EXAMPLES ................. . . . 19

9. COMPUTE TIMES ................. . . 20

10. SUBMITTING THE PRUGRAM . . . . . . . . . . . 22

10.1. Obtaining the Controllee and a Sample Input File.............. 22

10.2. Preparing the input file ........ 22

10.3. Running GFUN ............ 23

10.4. Running GFMOVEM ................ 25

APPENDIX B - EXAMPLES OF GFUN3D ON THE NMFE CDC $7690 \ldots 27$ APPENDIX C - AN ALTERNATIVE ITERATION TEChNIQUE FOR GFUN3D - - 29 


\section{ACKNOWL ELGMENTS}

This version of GFEi3n roulj not exist if C. h. Trowbridge, C. J. Coilie, N. J. Dis:arens, 1. J. Newman, J. Simkin, and A. G. Armstrong had not writter jif original. He hupe that nothing we have cone has caused orro:s ior which the; might receive unfair blame. We especially apureci, te the very kind comments Trowbridge has made about the a ternative iteration technique.

The initial canversi in of GFUN3D to a CDC 7600 was done before publication of $R L-76-0.9$ a see page 6.10). This version is based upon versions fon wail pinb. $\mathrm{c}$ s a made at LBL by $C$. W. Trowbridge and J. Colonias and a: : LL by 5 . $*$. T:owbridge and S. Sackett. Copier of those codes and rilans tur :pansion to larger problems were made available to us: he ar $\geq$ lrudebifci for this simflification of rur work.

K. h. Fong :I LII testrves special thanks. He not only discovered and ottaned the cojes ano plans mentioned above, but encouraged an! ir: ped throughiut the conversion. Further, he wrote a Iinear equations sys: is s.)lrer subroutine to be used for problems tro large for tie li: It has not be an used only because we have not yet needed larg:- nK.l' $s$ than LCM san handle.

T. A. Gut ir ard $R$. Ferguson of LlL have implemented the beam

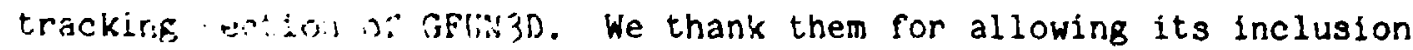
in this wasun anc rer tre many he!pful comments they have made.

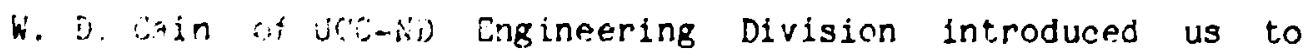


GFUN3D, taught us how to use an IBM/360 version, and encouraged us chroughout this work.

Janice $v$. Hughes has graciously helped us to rectify many mistakes through many draft versions of this report.

Finally, Y-K. M. Peng, R. A. Dory and J. Sheffield of ORNL Fusion Energy Division recognized the need for an MRFE version of GFUN'jD and provided support.

Thank you, all. 
CONVERSION AND IMPROVEAENT OF THE RUTHERFORD LABORATORY'S MAGNETOSTATIC COMPUIER CODE GFUN3D TO THE NMFECC CDC 7600

T. C. Tucker

\begin{abstract}
ABSThA:CT
We report the implementation of a version of the Rutherford Laboratory's magnetostatic computer code GFUN3I on the CDC 7600 at the National Magnetic Fusion Energy Computer Center. We include a new iteration technique which greatly increas - the probability of sonvergence and reduces computi on time by about 301 for calculations with nonlinear, ferromagnetic materials. We also discuss the use of GFUN3D on the NMFE network and present suggestions for future work. Appendix A consists of revisions to the GFUN3D User Guide (published by Rutherford Laboratory) which are necessary to use this version. Appendix $B$ contains input and output for sose sample calculations. Appendix $C$ is a detailed discussion of the old and new iteration techniques. (Appendices $A$ and $B$ are available in FILEM 27725 .GFUNFILE.)
\end{abstract}

\title{
HISTORY
}

The GFUN3D magnetostatics computer code $e^{1-3}$ is well known and widely used. One version has been operating on the Computer Sciences Division IBM/360/91 at ORNi since 1976. Long run times, slow turnaround, budget considerations, and limited precision (REAL4) led to a decision to implement GFUN3D on the NMFECC network. Initial work had been done on versions for a CDC 7600 by C. W. Trowbridge of Ruthorford Laboratory bitn J. Colonjas at Lawrence Berkeley Laboratory and $S$. Sackett at Lawrence .ivermore Laboratory. Neither of these versions could handie more than $35-40$ ifron el zments.

K. W. Fong of the MMFECC staff arranged for us to obtain source code for onth the LBL and LLL versions, together with notes and plans for more complote versions by Sackett and Colonias. Further disclissions with Fong, T. A. Cutler, and others at Livermore led to the 
decision to divide the implementation into two sections. Cutler was to implement the beam tracking and graphics sections, while T. C. Tucker, Oak Ridge, was to implement the major computation and data handling sections. Implementation was to be on the $\operatorname{CDC} 7600$ because initial work had been done on it and because the CRAY-I was not in operation at decision time.

\section{STATUS}

All of the projected work has not been finished, but a rery significant, improved versi.nn of GFUN3D is now operable on the MMFCCC 7600 (A machine). Use of this code is solicited and encouraged. It is not guaranteed to be error-free, but test runs have shcin general agreement with the IBM version at ORNL and the usable examples in the GFUN3D USER GUIDE.

The present NMFECC version of GFUN3D can calculate 170 iron elements in three dimensions or 255 in two. Graphics coding has not been implemented within GFUN3D, but some postprocessing capability exists on the 7600,4 and an interactive processor of DRAW and RECO commands exists for the DEC-10 at ORNL. 5 Nor is this version of GFUN3D interactive. It was decided that an interactive version for input might be appropriate for the input sections, but only with graphics included. Interactive problem-solving with GFUN3D was to be discouraged on the network. Non-interactive time-sharing for short runs and batch submission for longer runs are appropriate for GFUN3D on the NMPECC network. 
MECHANICS

The GFUN3D code for the MAFECC CDC 7600 has been implemented using the Los Alamos Scientific Laboratory FTN compiler (LASLFTK). This compiler was chosen over CHATR for its closer resemblance to "standard" FORTRA, its greater speed in compilation and executable code, and its compatibility with the Livermore Time Sharing System (LTSS). In addition, the Los Alamos Math Library (FTNMTH) available with LASLFTN contains a matrix package designed for large matrices stored in 7600 large core memory (LCM).

The UPDATE code was used to make changes in the GFIN3D source. This means that a record exists of all changes and the order in which they were made. If it is desired, any intermediate version can be recovered.

A three-level, eight-sagment overlay loading scheme was devised for GFUN3D on the A machine. This overlay scheme attempts to minimize thrashing of segments, especially for GETM, GFTB, and similar time-consuming commands. It aiso makes it possible to change required core dynamically. Thus, GFUN3D requires 130K (octal) words of SSM and $221 \mathrm{~K}$ (octal) words of LCM for everything but GETM commands which calculate 32 or more elements in three dimensions (47 or more in two). For larger cases, the additional core used is exactly the minimum needed. GFUN3D can be a good neighbor in time-sharing operation.

DIFFERENCES IN GFUN3D ON NMFECC 7600 AND ORNL 360

There are no basic differences between the GFUN3D implemented on the NMFECC 7600 and that on the ORNL IBM/360. There are differences in detall, however, primarily in input and output formats. Only in the 
addition of an alternative iteration scheme, including revised convergence criteria, is there any deviation from the basic GFUN3D algorithw.

There have been a number of changes in information printed and in its format. In all instances, the intent was to present additional information. Any data no longer presented at one particular place are automatically presented at another. For example, the comand PRINT originally caused two different descriptions of the iron elements. The first, which was an incomplete description for tetrahedral elements, also included the magnetization vectors, permeability, and susceptibility for the element. The second was a complete description of both prismatic and tetrahedral elements. In this version, the incomplete description has been replaced by the H-field (applied field) for the element and the susceptibility has been deleted. The complete iron description is still present.

An alternative iteration technique has been developed and coded into this version of GFUN3D. The original iteration scheme, which utilizes a relaxation factor, is still the default. The new technique must be requested by stating "CHIF=O" in the GETM command. This technique generally achieves convergence in about one-third fewer iterations than required by the original. The advantage is particularly pronounced when there is a wide range of inducing ( $H$ ) field over the Individual iron elements. Details of this technique are given in Appendix C. 


\section{VALIDATION}

The MMFE version of GFUN3D has been checked against results of the IBM/360 version at Oak Ridge National Laboratory and four of the examples in RL-76-029/A. Comparison to ORNL IBM/360 calculations shows agreement within tolerances which can be attributed to the differences in machine arithmetic precision, and sometimes with no observable differences.

Comparison to the examples in the GFUN3D USER MANUAL, al though more difficult, is alsc, generally satisfactory. In the examples, the presented input and output are not in a one-to-one relation. For instance, in 8.1 , the B-H data shown on page 8.2 do not correspond to Figure 8.1.2. If the data in Figure 8.1 .2 are used, one can calculate the equivalent of Figure 8.1.3. Further, Figure 8.1 .4 belongs to example 8.5 .

In Example 8.2, the 7600 version calculates a field about $1 \%$ nigher than that presented in Figures 8.2.5 - \&.2.8. The reason for this difference is unknown, but the ORNL IBM/360 version calculates a field essentially equal to that from the 7600 version.

Afain, in Example 8.3, the 7600 version does not agree with the plotted data in Figures 8.3.2 and 8.3.3 when the input shown are used. But, if the permeability is changed from 4669 to 4.669 , no observable differences exist.

Comparisons of Example 8.5 to results from the NMFE version are quite good. The plotted data shown in Figures $8.5 .3(a)$ and $9.5 .3(b)$ are reproduced to plot accuracy, and the data shown in Figure 8.1 .4 agree to printed accuracy with the interim listing at iceration 21. 
No compariscns were made to Examples 8.4 and 8.6 . In the case of 3.4, there were no numeric output data. In the case of 8.6 , the 200 iron elements exceed the capacity of this version of GFUN3D.

\section{GFUN3D ON THE MMFE NETWORK}

This version of GFUN3D is available for all MMFE wrork. A controllee, modified user's manual, sample calculations, and utility programs are contained in FILEM, user number 27725, directory GFUNFIIE.

A potential user must have a copy of the original GFUN3D USER GUIDE, PL-76-029/A. (That document can not be obtained from the implementor of the MMFE version.) File GFUP ..AAN contains Appendix $A$ of this report, the changes and differences applicable to the NMFE version. Note that GFUN.MAN contains upper and lower case, so a NETOUT must use option ULC.

\section{SUGGESTIONS FOR FUTURE WORK}

Further develcpment of GFUN3D on the MMFE network is expected to be limited. There are, however, three fields which might be considered. The first, and most obvious, is implementation on the Cray-I computer. The second is to introduce coding to eliminace the magietic eddies (looping) observed in a number of cases. The third is an attempt to include nonisotropic effects in the iron magnetization. Implementing GFUN3D on the Cray-I is not advisable at this time. There are two basic gains which might be achieved on the Cray. Decreasted run time is one. But, GPUN3D does not obviously lens 1 ise $1 f$ to vector calculation, so speed gains would be limited. Finther, the 7600 system currently has more tids, available than the Cray system, and 
turnaround is better on the 7600 . The second gain from a Cray version of GFUN3D would be the capability of solving larger problems. This gain is smaller than might be anticipated, however, with 275 iron elements ar approximate maximum. On the other hand, only minimal recodins would be required to increase the 7600 version from 170 to 190 elements in 3D, and a disk-using natrix solver would make 400 elements possible. (Such a matrix package näs been written, but not sheaked. Its inclusion in the 7600 version would be nontrivial.)

Coding to eliminate the nagnetic eddies should be considered. Ruthe:ford has introduced these shanges, but Joes not always use the modified version. 6 Although the coding changes are minor, additional computation time is not. Even so, the capability of eliminating the incorrect magnetizations hould be available to check the results fron the present code.

Inclusion of nonisotropic iron magnetization is not recommended at this time. There is insufficient data on actual iron behavior and there is nc obvjous, simple pro:edure which GFUN3D might use to approximate it. 
REFERENCES

1. GFUN: An Interactive Program as an Aid to Magnet Design, M. J. Newman, C. W. Trowbridge, I.. R. Turne:, Rutherford Laboratory, Chiltor., Didcot, Oxon, 0X 11 00X, England, Proceedings of the Fourth International Conference on Magnet Technology, Brookhaven National Laboratory, November 1972.

2. New Developments in the Magnet Design Computer Program SFUN3D, A.G.A.M. Armstrong, C. J. Collie, N. J. Diserens, M. J. li. wanan, J. Sizkin, C. W. Trowbritge, Hutherford Laboratc:y, Chilton, Didcot, Oxcn, OX11 OQX, England, Proceedings of the Fifth International Conference on Magnet Technology, Rome, April 1975.

3. GFUN3D USER GUIDE, A. G. Armstrong, C. J. Collie, N. J. Diserens, M. J. Newan, J. Simicin, C. W. Trowbridge, Rutherford Laboratory, Chilton, Didcot, Oxon, Oxil 00X, England, Report RL-76-029/A, November 1976.

4. Personal Communication, T. A. Cutler and R. Ferguson, Lawrince Livermore Laboratory.

5. Personal Communication, W. D. Cain, Engineering Division, Jak Ridge, Tennessee.

6. Personal Communication, C. W. Trowbridge, Rutherford Laboratory. 
APPENDIX A

GFUN3D NHFE 7600 USER'S GUIDE

This User's Guide contains only changes to or differences from the Rutherford saooratory GFIN3D USER GUIDE (RL-76-029/A). To facilitate use, it follows the format of the original USER GUIDE, and section and page numbers refer to that document. A copy of the latest version of this appendix is in FILEM as 27725 . GFUNFILE GFUN. MAN.

1. INTRODUCTIOA:

The package was modified for the MMFECC CDC 7600 computer by T. C. Tucker of ORNL and T. A. Cutler of LLL. It runs under the LTSS operating system in time-sharing or batch mode, but is not interactive.

2. INTEGRAL EQUATION METHOD

No changes.

3. THE USE OF INTERACTIVE GRAPHICS

No interactive graphics are available inr GFUN3D on the MMFE network. Non-interactive postprocessing graphics are being developed at Livermore, ${ }^{4}$ and an interactive preprocessivr for checking mojel definition is being developed on the DEC-10 system at ORNL. 5

There is a single controllee which does the work of both the "On-IIne (GFUN3D) and the off-line (GETM400)" programs referred to in $B,-76-029 / A$. 
4. STRUCtURE OF THE GFUN3D PROGRAM

The GFUN3D program on the MAFECC 7600 is a single code with overlays. It requires a minimum 221000 (octal) words LCM and about 130000 (octal) words of SSM. As the number of iron elements calculated increases above 31 in three dimensions (47 in two), LCM allocation is increased automatically to near the maximum possible at 170 elements in three dimensions or 255 in two.

5. UNITS

No changes.

G. COMMANDS AND PARAMETERS; TYPES, FORMATS, NOTES

\section{1. Command Names: List of Commands}

Cocumand input generally rollows the original sersion, including the footnotes for offline and $C D C$ versions. The input line contains a maximum of 72 characters, but the last "value" must not use column 72 . Only the comma indicating continuation can occupy column 72 . Numerical parameters cannot be in floating point (E) format.

The $C$ and $S$ commands (p. 6.1, bottom) are for interactive control. Since this is a non-interactive version, these commands are not implemented and should not be used.

The table of Commands, Punctions, and Parameters or, pages 6.2 and 6.? serves as an approximation only. Since graphics are not implemented, any command which plots, displays, etc., will not operate. (This version wil accept ant ignore any inoperable commands. An error message will not be issued in most cases.) The parameter list for some 
commands may not be accurate. Ir. particular, command parameters for the "direct access filing systew' differ as discussed below.

\subsection{DAAH: Enter E? ement Data}

\subsubsection{Generai Comments}

No changes.

\subsubsection{DRAH Comand for Specifying Coils}

On page 0.22 , a necessary parameter is omitted from the list for $E N D=6$, the cirsular arc corductor. The smitted parameter is $R 1$, which is erroneously labeled $k$ ir. Figure 6.2.18.

\subsubsection{DRAW Command for Specifying Iron}

Two iron SHAPE parameters to not operate as described in RL-76-029/A,, p. 6.39. FCr SHAP=-2, R1=0, the basic pentahedron is subd:vided into 4 tetrahedra. For $S H M P=-5, R 1=0$, the pentahedron is subdivided into 6 tetrahedra.

\subsection{MODI and MOVE: Modify and Move Elements}

No shanges.

\subsection{ERASE: Erase Elements}

No changes. 


\subsection{REC0: Reconstructs Pjetiure \\ Inoperative.}

\subsection{BHDA: Enter B-H Data}

The $C$ comand for removing the table entry (B-H pair) last stored is not operable. Numerical values of $B$ and $H$ must be in fixed point (F) format. To prevent $z$ divide-by-zero error, each B-value must be greater than the corresponding $H$-value. (An initial point $B=0, H=0$ is acceptable.) An "AA" in columns 1 and 2 signals the end of a B-H table.

When using the alternate iteration scheme, if the difference of $B$ and $H$ decreases in successive pairs of entries, saturation is presimer for the higher differtnce and all succeeding entries are ignored. This means that diamagnetic materials cannot be treated with this procedure.

\subsection{GETM: Magnetization Procedure}

The GETM procedure can calculate the magnetization for a maximum of 170 iron elements in three dimensions (255 in two). A maximum of 400 iron elements can be entered by DRAW commands. Any fron elements in excess of the 170 which can be calculated must be treated as "fixed" magnets by using the MODI and PIXI sommands.

Parameter NITD is not compulsory, as indicated on page 6.3. When data are entered by a READ command, NITD is included. This simplifies restarting an interrupted calculation. However, if a new model is to be specified by changing an old one through MODI, MOVE, etc., NITD=0 should be entered. 
The pirameter IT must be græater than NITD. The GETM section will a tom a maximus of Mil-dIT iterations.

$\therefore$ arzneter MPRI should iat be used. The run may abort with an outpit or raige error.

The initial permenbility, InU, need not be specified. Hith the ori sinal iteration. snece $z$ default of 1000 is available. The Alternate iceration prosed:is does not ise IMU.

The alternate iirrsion procedure is invoked by CHIF=0. This procedure generally requires one-third fewer iterations than the original method. Further, it has dchieved convergence in all cases tried, including wome in which the original failed.

The default vilue for TOLM is $10^{-6}$ rather than the $10^{-4}$ given on page 6.47, A new tolerance has been introduced for relative changes in $H, M$, and $X$ (susceptibility) of earh iron element. The default value for this tolerance is the square root o: TOLM, but it way be changed by TOLF, $=$

The original version of GFUN3D printed ar, intermediate listing of $H$ and $M$ for each element every 20 ite;ations, starting with iteration 1. Parameter LFRE has been introdiced to control the frequency of this listing. The value for LFRE may be 0 (never 11st), i (always list), or any other positive integer.

The parameter HEXT is used to specify a constant magnetic field in gauss in the $Y$ direction. 


\subsection{SETB}

Tt.e default value for parameter COMP is unkroin, but it is rot "HY" as stated on page 6.48. The field components, HX, HY, and $\mathrm{HZ}$ are always listed. Similarly, the default value of FINT seems to be variable. Field integrals are sometimes printed and sometimes omitted utién no FIMr is entered.

Parameters referring to plotting (ARROH, ERASE, etc.) are inoperative.

Wher a field is to be calculated at polar coordinate grid points, the plane of the polar grid must be specified by "PLANE".

\subsection{MAP}

Not operative.

6.10. HARM

The GETB command which precedes HARM must include parameter PLANE $=X Y$.

\subsection{File Manazement Commands}

GFUN3D on the MMFECC 7600 includes an optional filing system for model definitions and calculations. Use of this system is recomended. It allows saving magnetization data for later analysis, simplifies modifying model definitions, and provides a means of saving the latest iteration if time runs out during a batch run.

Since GFUN3D was designed for an IBM system, it is necessary to know the IBM nomenclature used in this section of RL-76-029/A. In that report, both "dataset" and "direct access filing system" refer to a 
large file wich corresponds to an LTSS disk file. "File" re.ers to a smaller collestion of data within the "dataset". (An approximate parallel is a :ile in a LIX library.) To miniaize confusion, we use "LiSS disk file" whenever the "dataset" is intended, and "file" whenever the snaller subset is meant.

The LTSS disk file must be defined and initizlized before it can be used by GFUN3D. This can be done easily by obtaining $z$ controllee from FILFM by

RDS 27725 .GFUNFILE SETFILE

and preparing a one-line input file named SETUPI. The file SETUPI must contain the desired rame of ycur LTSS disk file left-adjusted in columns 1-8. If a size other than one million ietal (262144 decimal) words is desired, that size is entered as a decimal number, right-adjusted in columns 11-20. (Omitting this number results in the default.) For example, to make a file named MYDATA which contains 256 segments of 512 words, SETUPI contains

$$
\begin{array}{lrr}
0 & 1 & 1 \\
\text { Col. } & 1234567890 & 1234567890 \\
\text { MYDATA } & 131072
\end{array}
$$

Then running SETFILE will define and initialize MYDATA, and also produce an output listing. SETUPO. This output file should consist of one line of text, about a dozen positive integers, three negative integers, and many zeroes. If SETUPO looks like this, the LTSS disk file is ready to use.

Command "LIST" has been completely changed. Parameter "DSN" has n.) effect since there is a single LTSS disk flle. Parameter "ID" has been replaced by an optional parameter "FL". If LIST is requested with no parameter, the entire directory wil]. be listed. Including 
"FL=value", where "value" is a string of one to four characters, lists all file names wich start with that character string.

Commands READ and DELE require special entry of a file name when a generation number is included. In particular, 5 to 8 characters must occur between "FL $="$ and the "," preceding the generation number. Trafling blanks are acceptable. For example

READ FL=JOE, 4

will fail, but

REA FL $=J 0 E b b, 4$ (where $b$ represents a space)

will retrieve JOE, generation 4 .

6.12. TRACK: Tracking of Charged Particles

$R \geqslant f e r e n c e$ to "C" and "S" commands under th: "PRINT" parameter should ve removed.

The "BEAM" command is inoperative.

\subsection{GETF: Magnetic Forces}

No changes.

6.14. INTE: Integration of Field Functions

Inoperative.

6.15. EIXI

No changes. 


\subsection{Other Commands}

There are four commands listed on pages 6.2 and 6.3 which are mentioned nowhere else in RL-76-029/A. These commands are discussed here.

FRAY is bas:.cally a graphics command, but GFUN3D refuses to run under sume conditions unless this command has been issued. A simple FRAM as the second line is sufficient.

PRIUT causes a fecailed pritting of the model including the description of coils; the corners, centroid, magnetization, and permeability of iron elements; and the B-H tables supplied. It is invoked automatically after every READ command.

CLEAR reinitializes the GFUN3D code. It allows stacking problems within a single controllee run.

END terninates a run.

7. OFF-LINE GETM

There is no separate "off-line" code.

\section{EXAMPLES}

We have calculated four of the $3 i x$ examples given in RL-76-029/A. Example 6.4 was not jone because nnly graphic output is shown in that report. Example 8,6 has 200 iron elements, exceeding the capacity of our version. Two of these examples have also been modified to illustrate the alternative convergence scheme.

In comparing results from these calculations to those presented in RL-76-029/A, some care must be exercised. In the sirst place, all figures show in that report do not result from the input data given, 
but are illustrative of a particular tme no... sut. For example, the B-H data shown as input for Example 8.1 includes pairs $(0,0)$, $(10000,6.99),(12000,9)$, etc. In Figure 8.1.2, however, the listing adds the pair $(8000,4.57)$ to the tab]s. If this point is added to the input, then the results shown in Figure 8.1 .3 are achieved (within machine precision). Further, Fijure 8.1 .4 actually comes from a run of Example 8.5.

A second caution about comparing to RL-76-029/A results concerns the scales on soxe graphs. The self-scaling algorithm used works correctly insofar as it includes all data points and little else. Unfortunately, the subdivisions are not always nice increments $(1,2$, cr 5 times a power of 10 is a nice increment), the number of minor subdivisions per major subdivision is variable (Figure 8.3 .2 has 7 minor/major on one axis), and the labels are often properly rounded but deceptive (.006 and .008 both rounded to .01$)$.

The input, output, and limited comments about the examples are presented in Appendix B. A more detalled discussion of the comparisons is in the body of this report.

\section{COMPUTE TIMES}

Execution time for GFUN varies widely with the problem. The most significant portions of the code for timing purposes are calculation of the magnetic field of the conductors at each iron element, definition of the matrix of interaction between iron elements, solition of the matrix equation, and calculation and analysis of the resultant field for the model. Data input and use of the LTSS disc file system usually requires less than 5 seconds. 
The time needed to define the matrix can be estimated by

$t_{d}=7 \times 10^{-4} n_{t}^{2.11} 2^{r}$

where

$t_{d}=$ time for matrix definition, seconds,

$n_{t}=$ total number of iron elements in model,

$r=$ number of iron symmetry reflecting planes.

For example, 50 iron elements with no symmetry planes require about 2.7 seconds, but 140 iron elements with 2 reflecting planes require about 95 seconds.

No simple estimating equation for the conductor field has been determined. The time should be proportional to the number of iron elements, the number of conductors, and the appropriate symmetry factors. The type of conductor model, however, appears to be most important, and no correlation has become apparent. Times observed for calculating the current field have ranged from virtually none to more than $40 \%$ of the matrix definition time.

The time required for matrix solution for one iteration can be estimated by

$$
t_{i}=7.4 \times 10^{-6} n_{c} 2.90
$$

where

$$
t_{1}=\text { time for one iteration, second } 3 \text {, }
$$

and

$$
n_{c}=\text { number of "free" iron elements. }
$$

Notice that the number of iron elements in the model $\left(n_{t}\right)$ may be greater than the number of "free" eiements $\left(n_{c}\right)$ being calculated. Approximate execution times are 0.63 seconds per iteration for 50 fiep elements and 21 seconds for 167 . The number of iterations required is 
quite variable, but one should estimate enoligh time for the maximum specified.

Again, there is no siaple formula for time needed to calculate the magnetic field for the model or to do an harmonic analysis or particle tracking calculation. It is best to save the magnetization on the LTSS disc file using "WRITE" and then do the calculations. Then: if insufficient time has been allowed for the field calcula'ions, the magnetization need not be recalcul.sted.

Overall execution time may run from less inail 30 seconds for 50 iron elements and a single solenois conductor to 30 minutes or more for 170 iron elements with multiple, variously shaped conductors.

10. SUBMITTING THE PROGRAM

19.1. Obtaining the Controllee ar.1 a Sample Input Fiie

The GFUN3D controllee, named GFUN, and a sample input file, GFUNIN, are obtained in FILEM by

RDS 27725 .GFUNPILE GPUN GPUNIN

This controllee will be updated as changes or correstions are made. Use the HOW command in FILEM to check the date the controllee was last changed.

\subsection{Preparing the Input File}

Input for the GRUN controllee must be in a file named GPUNIN. The first line of this file must contain the name of the LTSS disc file for the GPUN3D filing system in colinns 1-8 (see section 6.11) or be blank. Any other first line may cause an abnormal end. The second ine may or 
may not need to be a FRAM command. Therf is a possibility that some variables initialized by FRAM may sometimes be needed even though zraphics are not implemented. In any case, FRAM does no harm, and its presence on the second line is recommended. The order in the remaining input lines is restricted only in that a model must iirst be defined and thell magneti ced before fieid calculations can be made. The last line of the input file GFUNIN should always be the END command.

A sample GFUNIN is available as mentioned in the preceding section. It is suitable for a sample run requiring about 15 to $2 \hat{u}$ seconds cpu.

\subsection{Running GFUN}

The NMFECC 7600 version of GFIN3D can be rian in batch or timesharing (but noninteractive) mode. In either case the code generates and Jestrnys certain disc files, which the user may ignore. The names are chosen to be linique, thus they cannot affect any other files. Sowever, an output file named GFUNOUT is also written. It is essential that the user have no file which is to be kept named GFuNOUT because that $0:-$ file will be destroyed when GFUN is staried.

In timesharing mode, one prepares the input file GFUNIN, enters GEUN / $\mathrm{t} v$

from the keyboard, and when the code finishes, does something with GFUNOUT. Note that GFUNIN and GFUNOUT are special names, and hence only one controllee car. be run at any time by a single user.

In batch mode, it is recommended that the controllee always be read from 27725 .GFUNFILE. This assures the latest version. Multiple batch iuns naturally require multiple input files, so remember to 
change the name to GFUNIN before executing GFUN. The first batch operation after GFUN should be a FILEA replace of the Cr N3D filing system LTSS disc file. If an iteration runs ou: of time, the last iteration will be written to the I.TSS disc file (see Sec. 6.11); but, if that file is not written to FILES, it will be lost when batch cleans up.

There are two sources of potential trouble for the standard, printed output from GFUN3D batch uns. This out.put appears as a family of files starting with GFUNOUT. One must ensure that all members of the family are used. If this output is to be kept in FILEM, one must ensure it does not conflict with some other sec. The simplest solution is to include the following COSMOS instructions:

- FILEM RDS .GFUNFILE COMPACT

- COMPACT GFUNOUT NEWNAME

This will replace all members of the GFUNOUT family by a single file called NEWNAME.

Another problem in batch runs arises from the possible parallel execution of two or more GFUN jobs. If the two jobs write into the same LTSS disk file, the second job's replacing the file in FILEM will overwrite the output from the first. The solution is to use multiple LTSS disk files for batch runs which may run simultaneously. Such files do not need to be very large. An interactive utility program, GPMOVEM, has been written to facilitate ihe necessary data manipulation. Given two LTSS disk files, GFMUVE can copy a problem file from one LISS disk file to another, list the contents, and delete spectfied files. Thus many small LTSS disk flles can be used for a number of batch runs and the results transferred to a master for 
permanent sturage. Instructions for GFMOVEY are in the following section.

\subsection{Running Getovar}

An interactive program has been written for manipulating LTSS disk files for the GFUN direct access filing system. The controllce GrMOVEM can be obtained by

FILEM RDS 27725 .GFUNF ILE GFMOVEM .

The commands for GFMOVEM are analogous or identical to the GFUN3D file management commands (Sect. 6.11). GFMOVEM can operate on one or two LTSS disk files at once. These files and their use are speoified by the following commands:

FROM DS $=$ filenamei

INTO DS $=\underline{\text { filenamez }}$

The commands READ, URITE, and DELETE operate upen the grammatically correct file. They READ or DELETE FROM, but WRITE INTO. Commands LIST and ROOMAC operate on both files; but, if only one is specified, the code does not object.

One runs GFMOVEM by entering

GFMOVEM $/ \mathrm{t} v$

and responding to the prompt with a command. The usual END command will terminate the run.

Two further commands have been implemented in GFMOVEM. Tise first of these is

SOPY FL=name, sen .

This results in a READ of name, gen FROM Lllenamel followed by a WRITE of name INTO Cllename?. Note well, however, that the generation number 
may be changed. (The last paragraph of Sec. 6.11, cencerning the number of characters in name, applies here also.) The command MOVE FL=name, Ben

causes the READ and YRITE of COPY, followed by a DELETE of name, gen FROM filenamel.

Commands COPY and MOVE are convenient, but name is unchanged. If one wishes to change the name of a set of Jata, then READ and WRITE are the appropriate commands.

The LTSS disk ililes in use can be changed at any time by issuing a FROM or INTO. The only restriction is that a single LTSS disk file cannot be opened for both. 
APPENDIX .9

EXAMPLES OF GFIN3D ON THE MTE CDC 7600

This appendix is published as a microfiche which is enclosed at the back of the report.

The examples in this appendix are also available in FILEM as, for example, 27725 .GFUNFILE EiAMPLE8.1. The complete list of names san be obtained by LST 27725 .GFUNFILE ALWITH. EXAM. 


\section{APPENDIX C \\ AN ALTERHATIVE ITERATION TECHNIQUE FOR GFUN3D}

We have developed an alternative iteration technique for GFUN3D on the MAFE 7600. The original technique is o.ren slow, sometimes fails to converge, and has incorrectly indicated convergence on occasion. The new technique generally needs one-third fewer iterations and has always converged, even on cases for which the original method fails.

The basic equation of GFUN3D is

$$
H_{k}=C_{k}+\sum_{j} G_{j} k^{M} \mathbf{j}
$$

where

$H_{k}$ is the total magnetic intensity at point $k$,

$C_{k}$ is the magnetic intensity at $k$ caused by currents and fixed
fields,

$M_{\mathfrak{j}}$ is the constant, magnetization throughout iron element $\mathfrak{j}$.

and

$G_{j k} k_{j}$ is the magnetic intensity at $k$ caused by element $j$. (Variables $H_{k}, C_{k}$, and $M_{j}$ are m-dimensional vectors and $G_{j k}$ is an $m \times m$ matrix, but it is sufficient to consider them as scalars for this discussion.) However, iron magnetization, $M$, is a function of the applied magnetic intensity, $H$, and way be expressed by

$$
H=X \cdot H
$$

where $X$ is the iron susceptibility, not generally a constant. In GFUN3D, if point $k$ in (1) is interpreted as a representative point for iron element $k$, then (1) and (2) lead to

$$
\sum_{j k} G_{j k} M_{j}+\left(G_{k k}-1 / x_{k}\right) M_{k}=-C_{k}
$$

Thus, with a known susceptibility for each iron element, calculation of the magnetizaticns is a linear problem. If susceptibility is not 
known, GFUN3D assumes a value for each element and solves for the magnetizations, iterating with successive assumptions until some convergence for consistency criterion is satisfied. The procedure for choosing the successive values for $X$ and the convergence criterion make up the iteration technique.

In the original GFUN3D, the iteration technique consists of the following steps:

1. Assume a single susceptibility, $X_{k, 0}$, for all elements (notice that a second subscript now denotes the iteration number.)

2. Solve the system of equations (3) for $M_{k, i}$ using $X_{k, 1-1}$.

3. Calculate the total field $H_{t, i}$ at some test point $t$.

4. If $\left|H_{t, i}-H_{t, i-1}\right| S \varepsilon_{t} H_{t, i}$ where $\varepsilon_{t}$ is a specified tolerance, presume convergence and stop iteration. Dtherwise, proceed to step 5. $X_{k, i}=M\left(H_{k, i}\right) / H_{k, i}$.

5. Caj culate $H_{k, i}=M_{k, i} / X_{k, 1-1}$.

6. Find $M\left(H_{K_{2}}\right)$ frcm tabular input data giving $B=M+H$ as a function of $\mathrm{H}$, and define

$$
X_{k, 1}=M\left(H_{k, i}\right) / H_{k, i} \text {. }
$$

7. Determine $x_{k, i}$ from

$$
x_{k, 1}=w x_{k, 1}^{k}+(1-w) x_{c, 1-1},
$$

where $w$ is the relaxation factos (input parameter CHIF), and iterate from step 2 .

This technique can fail completely when $w>1$ and $x_{k, 1}<$ $(w-1) X_{k, 1} / w$. The result is a negative susceptibility, an impossibility for ferromagnetic matericis. The iteration can sometimes recover fror. such a situation, but often cannot. Again, the singie point convergence test in step 4 can be and has been passed accidentaliy with susceptibilities and magnetizations nowhere close to true solution values. Finally, the $\chi^{*}$ determined in step 6 often approaches the 
solution asymptotically (especially for iron elements near saturation), causing a slow rate of convergence.

There are a number of ways to avoid false convergence after the fact. (First, of course, it must be recognized. This is not always trivial.) Changing the initial susceptibility by specifying $a$ different input value for IMU may work. Choosing a different test point is another way. Unfortunately, the only method of assuring convergence with the original technique is to choose a relaxation factor no greater than 1. This choice necessarily limits the rate of 3symptotic approach and slows convergence. Thus, two of the problems have opposing solutions.

Trowbridge $^{2}$ has recognized the flaws in the original iteration technique. He proposed a secant me hod for accelerating convergence. Difficulties in implementation and higher priorities for other work lefl the secant method in the proposal stage.

We met all three convergence problems in calculating ISX magnetization using GFUN3D. We wanted a new iteration procedure which would assure convergence of each iron element as well as the external fleld. We wished to avoid initial guesses and user intervention, usually an after-the-fact correction. We believed that the iteration history should contain information which could be used to accelerate convergence. Starting from these desires and beliefs, we made a number of experimental changes in the GFUN3D iteration.

Standard techniques for accelerating convergerice, e.g. Aitken's $\delta^{2}$ process, did not work. Attempts to automate the cholce of relaxation factor sailed. But a number of procedures were sound which 
worked well under certain circumstances. A selestive combination of these makes up the new iteration technique.

The new iteration technique consists of the following steps:

1. Find the entry in the input B-H table with maximum difference $B$ minus $H$. This is taken as the saturation point. (Note that this presumption eliminates diamagnetic materials.)

2. Calculate $H_{k, 0}$ at each element assuming susceptibility $X_{k}=$ 0 , and deterwine the corresponding $k_{k, 0}$ from the input data using linear interpolation. Assuse infitial, individual susceptibilities

$$
X_{k, 0}=\mathbf{H}_{k, o} / H_{k, 0}
$$

3. Solve the system of Eqs. (3) for $M_{k, i}$ using $X_{k, i-1}$. Calculate $H_{k, i}=M_{k, i} / X_{k, i-1}$.

4. Find the "near point", $\left(\mathrm{HN}_{k}, \mathrm{MN}_{k}\right)$, which minimizes

$$
\left(\frac{\mathrm{HN}_{\mathrm{k}}}{\mathrm{H}_{\mathrm{k}, \mathrm{I}}}-1\right)^{2}+\left(\frac{\mathrm{MN}_{\mathrm{k}}}{\mathrm{H}_{\mathrm{K}, \mathrm{i}}}-1\right)^{2}
$$

for each iron elemeht.

5. Find all intersections $\left(\mathrm{HI}_{\mathrm{k}}, \mathrm{MI}_{\mathrm{k}}\right)$ of the line through $\left(\mathrm{H}_{\mathrm{k}, 1}\right.$, $\left.M_{k, i}\right)$ and $\left(H_{k, 1-1}, M_{k, 1-1}\right)$ and the curve defined by linear interpolation of the input table.

6. If any $\left(H I_{k}, M I_{k}\right)$ points interpolate $\left(H_{k, 1}, M_{k, 1}\right)-\left(H_{k, 1-1}\right.$, $\left.M_{k, 1-1}\right)$ use the measure in step 4 to find the nearest ( $\mathrm{HI}_{k}$, $\left.M I_{k}\right)$. Then the new estimate for susceptibility is $X_{k, 1}=$ $M I_{k} / \mathrm{HI}_{\mathbf{k}}$. Proceed to step 9. 
7. If any $\left(H I_{k}, M I_{k}\right)$ points exist such that

$$
0<\frac{\left(H_{k, i}-H I_{k}\right)}{\left(H_{k, i}-H N_{k}\right)}<r
$$

and

$$
\frac{\left|M_{k, i}-M I_{k}\right|}{\left|M_{k, i}-M N_{k}\right|}<r
$$

where $r$ is an arbitrary constant, choose the nearest as $\left(\mathrm{HI}_{k}^{*}\right.$, $M I_{k}^{*}$ ). Set $X_{k, i}=M I_{k}^{*} / I_{k}$ and proceed to step 9 .

8. If $X_{k, i}$ is not set in step 6 or 7 , then $X_{k, i}=M N_{k} / H_{k}$.

9. Calculate the total fiel,i $\mathrm{H}_{t, i}$ at some test point $t$.

$$
\begin{aligned}
\text { 10. If }\left|H_{t, i}-H_{t, i-1}\right| \leq \varepsilon_{t} \cdot H_{t, i}, \\
\left|H_{k, i}-H_{k, i-1}\right| \leq \varepsilon_{e} \cdot H_{k, i}, \\
\left|M_{k, i}-H_{k, i-1}\right| \leq \varepsilon_{e} \cdot M_{k, i},
\end{aligned}
$$

and

$$
\left|x_{k, i}-x_{k, i-1}\right| \leq \varepsilon_{e} \cdot x_{k, i}
$$

for all iron elements, where $\varepsilon_{t}$ and $\varepsilon_{e}$ are specified tolerances, presume convergence and stop iteration. If any of the tests is fafled, iterate from step 3.

The assumption of a saturation point in step 1 simplifies data input and provides a means for avoiding incorrest magnetization for $H$ fields larger than expected. Step 2 eliminates initial guesses of iron permeability and provides individual values for each fron element. The near point evaluation of steps 4 and 8 finds a susceptibility very 
close to that wich is found by the origial technique as an iron element approaches saturation. When an element is in the high susseptibility portion of the curve, the near point selection essentially inverts the original scheme by finding $H$ as a function of M. This works well because the $1 / X$ term of Eq. 3 becomes negligible. In the region of the knee, the near point makes a smooth transition.

Steps 5, 6, and 7 essentially perform "regula falsi" when possible and otherwise use Newton's method with a first difference approximation to the derivative. The arbitrary constant, $r$, in step 7 is intended to prevent gross changes which perturb other elements too much. In our inftial runs, $r$ was set at 2 . Since convergence has been achieved rapidly with this value, no tuning has been attempted. The other restriction of step 7 (that $H_{k, i}-H_{k}$ have the same sign as $H_{k, i}$ $\mathrm{HN}_{\mathrm{K}}$ ) was found to be essential.

The tests of step 10 assure that all iron elements are converged. All of $H, M$, and $X$ must be checked because there are regions of the magnetization curve in which any one may be virtually constant wile the other two change. We have set $\varepsilon_{e}=\sqrt{\varepsilon_{t}}$ as a default. Limited experimen:s have shown little to be gained in speed or accuracy by using a different value.

Using this teohnique in the MMPE CDC 7600 version of GPUN3D increases the time per fteration by less than $5 \%$, but generally cuts the number of iterations by about one-third, The net gain is a decrease in iteration time on the order of 301 . More important is that this technique has always converged. 